\title{
NEW TECHNIQUES FOR COMPUTING GEOMETRIC INDEX
}

\author{
K.B. ANDRIST, D.J. GARITY, D.D. REPOVŠ, AND D.G. WRIGHT \\ Dedicated to Sibe Mardešić (1927-2016)
}

\begin{abstract}
We introduce new general techniques for computing the geometric index of a link $L$ in the interior of a solid torus $T$. These techniques simplify and unify previous ad hoc methods used to compute the geometric index in specific examples and allow a simple computation of geometric index for new examples where the index was not previously known. The geometric index measures the minimum number of times any meridional disc of $T$ must intersect $L$. It is related to the algebraic index in the sense that adding up signed intersections of an interior simple closed curve $C$ in $T$ with a meridional disc gives \pm the algebraic index of $C$ in $T$. One key idea is introducing the notion of geometric index for solid chambers of the form $B^{2} \times I$ in $T$. We prove that if a solid torus can be divided into solid chambers by meridional discs in a specific (and often easy to obtain) way, then the geometric index can be easily computed.
\end{abstract}

\section{INTRODUCTION}

The geometric index of a link $L$ in the interior of a solid torus $T$ measures the minimum number of times any meridional disc of $T$ must intersect $L$. The geometric index of $L$ in $T$ often seems intuitively obvious, but it is surprisingly difficult to prove that intuition corresponds to the actual index. See for example the links in Figures 2, 3, and 4. Various ad hoc methods were used to compute the geometric index of the links in Figure 4 in $[\mathrm{AW}]$ and [GRW17], but these methods do not apply to links in general. The method described in this paper encompasses the ad hoc arguments used in those papers and applies more generally to links in a solid torus.

In this paper we introduce new techniques for computing the geometric index of $L$ in $T$. The strategy is to break up the solid torus into chambers $C_{i}$ of the form $B^{2} \times I$ by a carefully selected collection of meridional discs, and to analyze the part of the link $L$ in each $C_{i}$. The chambers can in practice be chosen so that all but two of the segments in $C_{i}$ meet each end

2010 Mathematics Subject Classification. Primary 57M25, 54E45, 54F65; Secondary 57N75, 57M30, $57 \mathrm{~N} 10$.

Key words and phrases. algebraic index, geometric index, Whitehead link, Bing link, McMillan link, Gabai link, Antoine link.

The authors would like to thank the referee for helpful suggestions and for pointing out useful clarifications to include. The authors were supported in part by the Slovenian Research Agency grant BI-US/15-16-029. The second author was supported in part by the National Science Foundation grant DMS0453304. The second and fourth authors were supported in part by the National Science Foundation grant DMS0707489. The third author was supported in part by the Slovenian Research Agency grants P1-0292 and J1-6721. 
of $C_{i}$ in a single point. This can be done by isolating turning points or pairs of turning points in components of $L$ in separate chambers. This method holds for the links in the literature that we have examined. Each meridional disc of $C_{i}$ must intersect such a segment. This reduces the analysis in each such chamber to determining whether an arc with both endpoints in one end of the chamber links an arc with both endpoints in the other end. To make this precise, we define the geometric index of $L \cap C_{i}$ in $C_{i}$. If each such geometric index is $n$, and if each meridional disc in the chosen collection intersects $L$ transversely in $n$ points, then we show that the geometric index of $L$ in $T$ is $n$.

This is stated precisely in Theorem 4.4. The three corollaries that follow from this theorem give various methods for computing the geometric index from information about the chambers. One might think a simpler result could be obtained by not requiring that each meridional disc in the collection intersects $L$ in exactly $n$ points, and that one could simply take the minimum geometric index of the link in the collection of chambers. The example in Figure 1 shows that this is not the case.

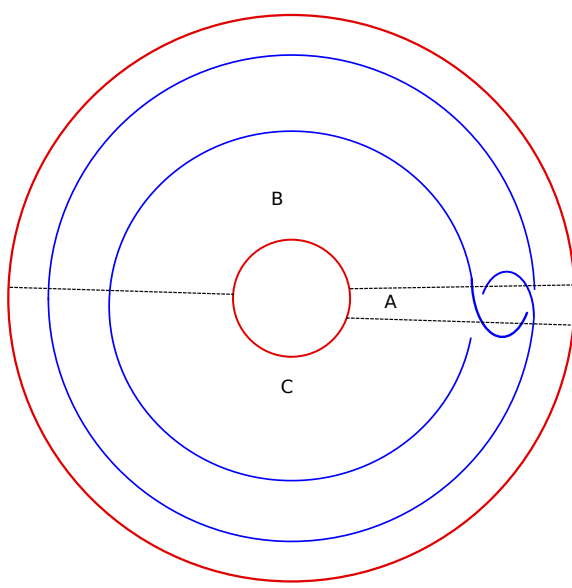

(a) Index 2

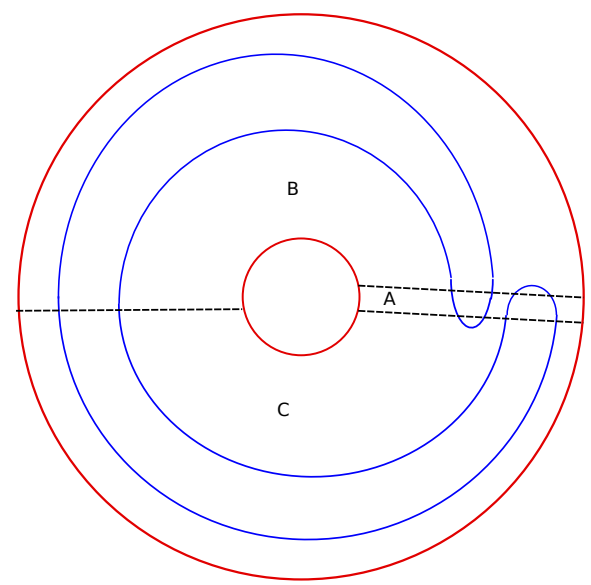

(b) Index 0

FiguRE 1. Chambers with Same Indices

In each subfigure, Chamber A has geometric index 4 and Chambers B and $\mathrm{C}$ have geometric index 2. But the overall geometric indices of the two examples differ.

A specific case of this result for certain special links in the setting of only one meridional disc appears in $[\mathrm{AW}]$. Another specific simple case of this result for one specialized type of link and two meridional discs appears in [GRW17]. Section 2 contains terminology, definitions and basic results about geometric and algebraic indices. This section also indicates how geometric index computations can be extremely useful in proving certain geometric results. Section 3 defines geometric index for chambers of the form $B^{2} \times I$ and computes the geometric index in a few special cases. Section 4 contains the proof of Theorem 4.4 and three corollaries. Section 5 contains examples of applications of the main results. 


\section{Definitions and Results about Geometric and Algebraic Indices}

If $S$ is a solid torus embedded in another solid torus $T$, the algebraic index of $S$ in $T$, $a(S, T)$, is defined to be $|\alpha|$, where $\alpha$ is the integer in $H_{1}(T)$ represented by the center line of $S$. Algebraic index is multiplicative, so that if $S \subset T \subset U$ are solid tori, the algebraic index of $S$ in $U$ is the product of the algebraic index of $S$ in $T$ with the algebraic index of $T$ in $U$. Note that the algebraic index of a Whitehead link in the torus containing it is 0 , as is the algebraic index of each component of a Bing link. See Figure 3.

Schubert introduced the notion of geometric index in [Sch53]. If $K$ is a link in the interior of a solid torus $T$, then we denote the geometric index of $K$ in $T$ by $N(K, T)$. The geometric index is the minimum, over all meridional discs $D$ of $T$, of $|K \cap D|$. A core of a solid torus $T$ in 3-space is a simple closed curve $J$ such that $T$ is a regular neighborhood of $J$. Likewise, a core for a finite union of disjoint solid tori is a link consisting of one core of each of the solid tori. If $T$ is a solid torus and $M$ is a finite union of disjoint solid tori so that $M \subset$ Int $T$, then the geometric index $N(M, T)$ of $M$ in $T$ is $N(K, T)$, where $K$ is a core of $M$.

Figure 2 indicates a collection of new examples. Each circled part of the inner link in Figure 2(a) can be replaced by one of the patterns in Figure 2(b) to produce many different examples. These examples do not fit into any previous class of examples such as the Gabai or McMillan links in [GRW17]. More complicated links can also be obtained by additional varying of the linking or winding pattern of the strands as one progresses out from the center of the diagram.

It is clear that the algebraic index of the inner link in Figure 2(a) is 0. Our techniques show that the geometric index is 8 for each example. See Section 5 .

The geometric indices of the links in Figure 3 are all two. The algebraic index of the Whitehead Link in Figure 3(a) is zero as is the algebraic index of each component in Figure $3(\mathrm{~b})$ and Figure 3(c). The algebraic index of the link in Figure 3(d) is two. The geometric indices of the links in Figure 4 are indicated in the figure. More details will be provided in Section 5. See [GRW17] for more discussion of the Gabai and McMillan links in Figure 4.

Remark 2.1. It is well known that the algebraic index of a simple closed curve $J$ in a solid torus $T$ can be computed as follows. Choose a meridional disc $D$ that intersects $J$ transversely. Assign a positive and a negative side to $D$ in $T$ so that a simple closed curve passing through $D$ once, going from the negative to the positive side, has algebraic index 1 in $T$. Assign a \pm 1 to each intersection of $J$ with $D$ corresponding to passing from the negative to the positive or from the positive to the negative side. Then the algebraic index of $J$ in $T$ is the absolute value of the sum of the numbers assigned to each intersection of $J$ with $D$. In particular, the algebraic and geometric indices have the same parity.

Remark 2.2. Note that the previous remark immediately implies that the geometric index is greater than or equal to the algebraic index.

The next few results follow from Schubert [Sch53] and regular neighborhood theory [RS82]. They indicate the usefulness of being able to compute geometric index. For more details 


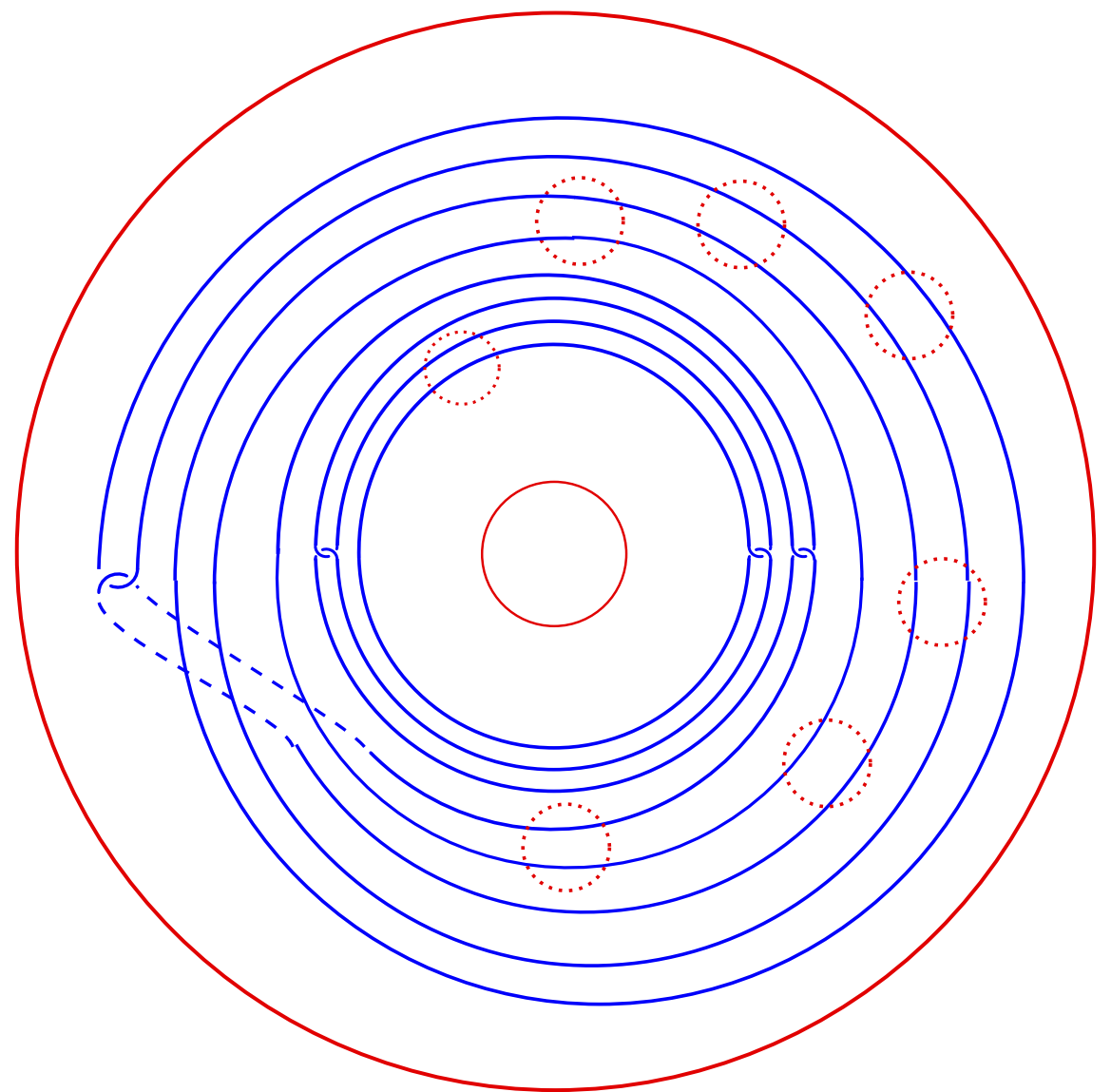

(a) Complicated Link(s)
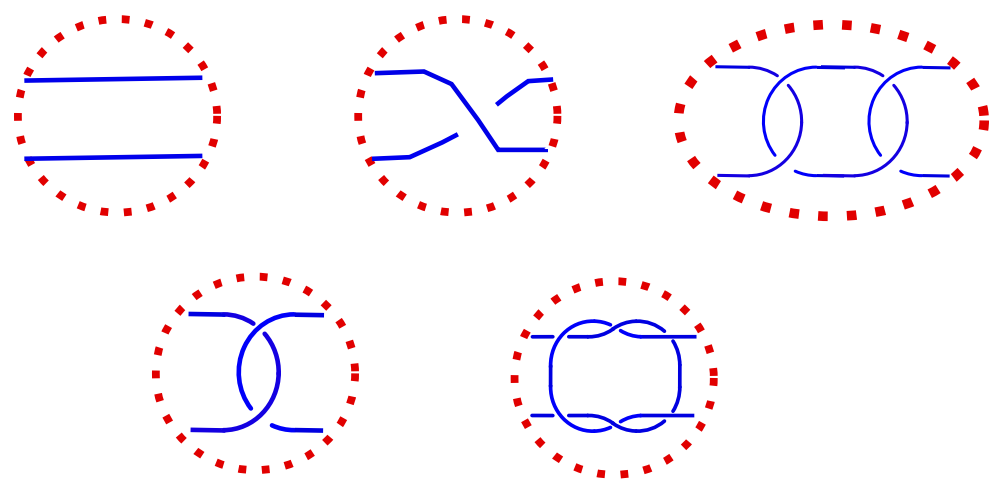

(b) Replacement Strands

FiguRE 2. Some Complicated Links

on the proofs of these lemmas, see Section 3 in [GRWŽ11]. We include a proof of the third lemma to give an indication of the techniques used. 


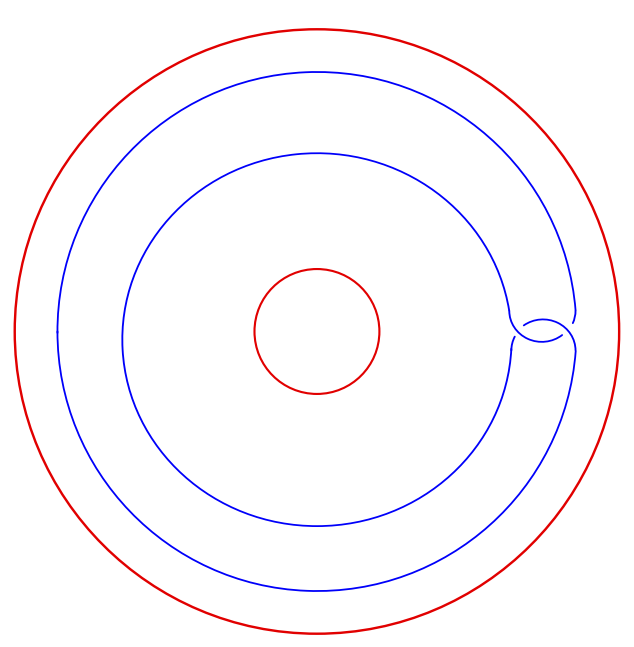

(a) Whitehead Link

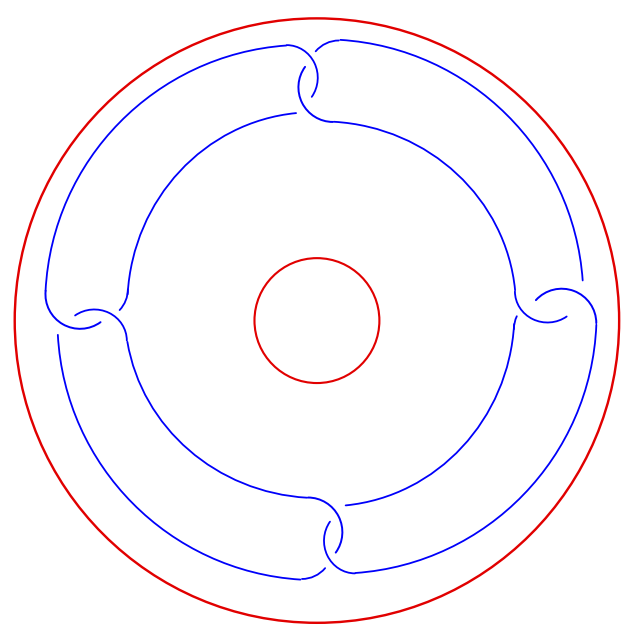

(c) Antoine Link

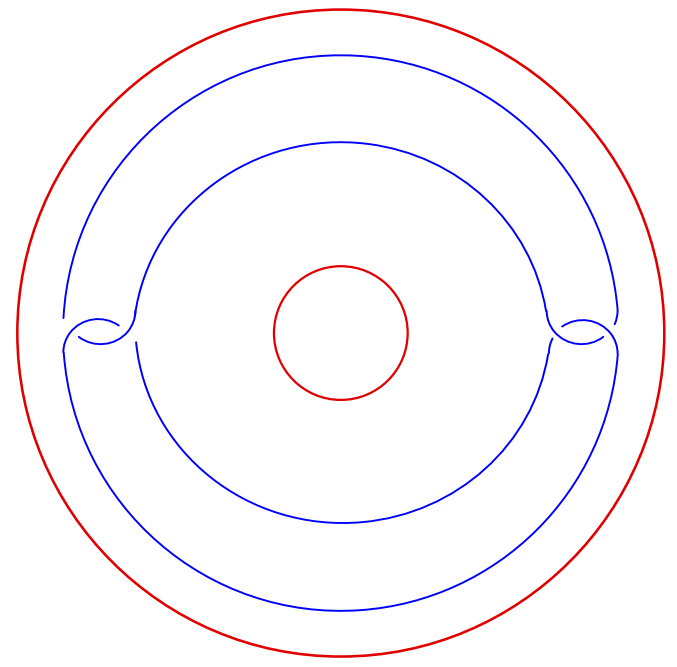

(b) Bing Link

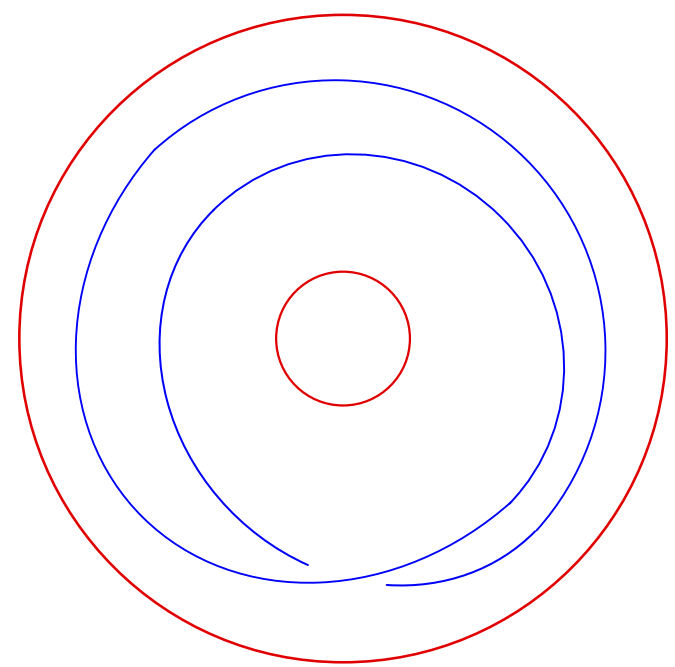

(d) Algebraic Index 2

FIGURE 3. Links with Geometric Index 2

Lemma 2.3. Let $T_{0}$ and $T_{1}$ be unknotted solid tori in $S^{3}$ with $T_{0} \subset \operatorname{Int} T_{1}$ and $N\left(T_{0}, T_{1}\right)=1$. Then $\partial T_{0}$ and $\partial T_{1}$ are parallel; i.e., the manifold $T_{1}-I n t T_{0}$ is homeomorphic to $\partial T_{0} \times I$ where $I$ is the closed unit interval $[0,1]$.

Lemma 2.4. Let $T_{0}$ be a finite union of disjoint solid tori in $S^{3}$. Let $T_{1}$ and $T_{2}$ be solid tori so that $T_{0} \subset$ Int $T_{1}$ and $T_{1} \subset$ Int $T_{2}$. Then $N\left(T_{0}, T_{2}\right)=N\left(T_{0}, T_{1}\right) \cdot N\left(T_{1}, T_{2}\right)$.

Lemma 2.5. Let $T$ be a solid torus in $S^{3}$ and let $T_{1}, \ldots T_{k}$ be disjoint unknotted solid tori in $T$, each of geometric index 0 in $T$. Then the geometric index of $\cup_{i=1}^{k} T_{i}$ in $T$ is even.

Proof. If the geometric index were odd, then there would be a meridional disc $D$ of $T$ that would intersect the cores of $\cup_{i=1}^{k} T_{i}$ transversally an odd number of times. This would mean 


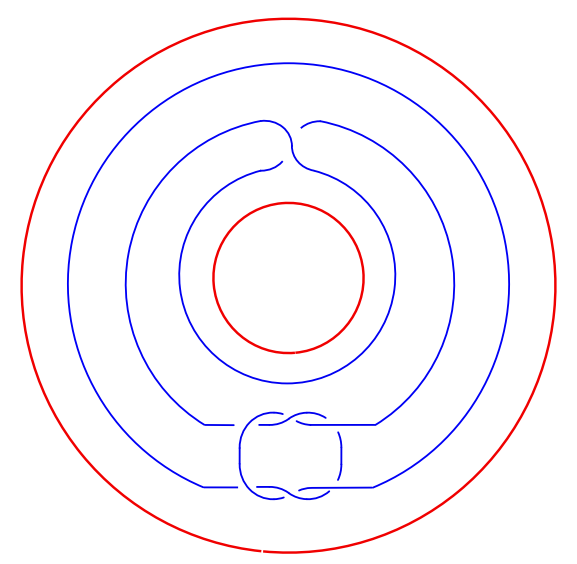

(a) Knotted Link - Index 3

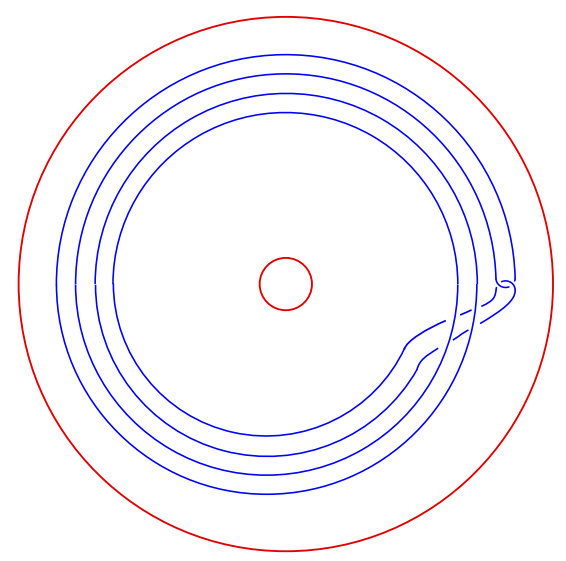

(b) McMillan Link - Index 4

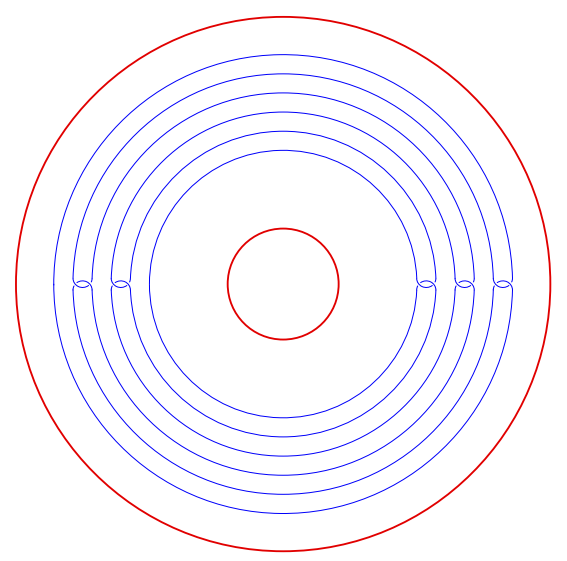

(c) Gabai Link - Index 6

Figure 4. Additional Links

that $D$ must intersect the core of some $T_{i}$ an odd number of times. But if a meridional disc of $T$ intersects a simple closed curve $J$ transversally an odd number of times, the algebraic index of $J$ in $T$ is odd by Remark 2.1, and so $J$ is essential in $T$. However, for each $i$, the core of $T_{i}$ is inessential because it lies in a ball in $T$.

The next two results make use of the material on geometric index to determine when the boundaries of certain tori are parallel as a further illustration of the use of geometric index. See [Wri89] for details. We include the proof of the first theorem to further indicate the techniques used.

Theorem 2.6. Let $W$ be torus in a solid torus $T$ in $S^{3}$ with the core of $W$ forming a Whitehead link (as in Figure 3(a)) in $T$. If $T^{\prime} \subset T$ is a solid unknotted torus whose boundary separates $\partial W$ from $\partial T$, then $\partial T^{\prime}$ is parallel to either $\partial W$ or $\partial T$. 
Proof. Since $\partial T^{\prime}$ separates $\partial W$ from $\partial T$, we have $W \subset \operatorname{Int} T^{\prime}$ and $T^{\prime} \subset$ IntT. Since $N\left(W, T^{\prime}\right) \cdot N\left(T^{\prime}, T\right)=N(W, T)=2$, either $N\left(W, T^{\prime}\right)=1$ or $N\left(T^{\prime}, T\right)=1$. The conclusion now follows from Lemma 2.3.

Theorem 2.7. Let $F_{1} \cup F_{2}$ be a pair of disjoint tori in a solid torus $T$ in $S^{3}$, with the cores of $F_{1}$ and $F_{2}$ forming a Bing link (as in Figure 3(b)) in $T$. If $S$ is the boundary of a solid unknotted torus that separates $\partial F_{1} \cup \partial F_{2} \cup \partial T$, then $S$ is parallel to one of $\partial F_{1}, \partial F_{2}, \partial T$.

\section{Geometric Index for Chambers}

In this section, we introduce the concept of geometric index for chambers of the form $B^{2} \times I$, formed by pairwise disjoint meridional discs in a solid torus.

A chamber $C$ is a space homeomorphic to $B^{2} \times I$. The top of the chamber, $C_{t}$, corresponds to $B^{2} \times\{1\}$ and the bottom of the chamber, $C_{b}$, corresponds to $B^{2} \times\{0\}$. We think of the chamber $C$ as the region between two meridional discs in a solid torus, with the meridional discs corresponding to $C_{t}$ and $C_{b}$. An interior meridional disc $M$ in such a chamber $C$ is a disc in $C-\left(C_{t} \cup C_{b}\right)$, where $\partial M$ is essential in $\partial C-\left(C_{t} \cup C_{b}\right)$. Let $L$ be a collection of arcs and simple closed curves in a chamber $C$ so that each arc has its endpoints in $C_{t} \cup C_{b}$ and so that each simple closed curve is in $C-\partial C$. The geometric index of $L$ in $C, N(L, C)$, is the minimum of $|L \cap M|$ over all interior meridional discs $M$ of $C$.

Consider a link $L=A \cup B \cup S$ where $A$ is an arc with endpoints in $C_{t}, B$ is an arc with endpoints in $C_{b}$, and $S$ is a possibly empty union of circles. The link $L$ forms a clasp in $C$ if the geometric index of $L$ in $C$ is two. A spanning arc $\mathrm{A}$ in $C$ is an arc with one endpoint in $C_{t}$, and the other endpoint in $C_{b}$. See Figure 5 for some examples.

Lemma 3.1. The Whitehead clasp, the Square Knot clasp, and the Antoine clasp (pictured in Figure 5(b), Figure 5(c), and Figure 5(e)) have geometric index 2 in the indicated chambers. Each spanning arc in a chamber has geometric index 1 in that chamber.

Proof. Any interior meridional disc must intersect a spanning arc, even if the arc is knotted in the chamber. To see this, note that any meridional disc divides the chamber into two components with $C_{b}$ in one component and $C_{t}$ in the other. If the meridional disc misses the arc completely, then the arc is contained in one of the two components which cannot happen.

For the Whitehead clasp, consider Figure 6. Let $D$ be a disc in the chamber with boundary $A$ together with an arc in one end of the chamber that joins the two points of $A$ in that end. There is no loss of generality in only considering interior meridional discs that intersect $A \cup B$ transversely since non-transverse intersections can be removed by a small general position move which only decreases the number of intersections. By an argument similar to that in the proof of Lemma 2.5, every such meridional disc must intersect each of $A$ and $B$ an even number of times. There is clearly an interior meridional disc that intersects $A \cup B$ twice, so we must only eliminate the possibility of no intersections.

Suppose $M$ is an interior meridional disc that misses $A \cup B$. By a general position adjustment away from $A \cup B$, we can assume that $M$ intersects the disc $D$ in a finite number of simple 


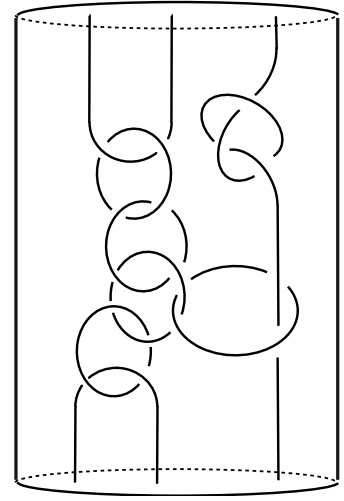

(a) Link in Chamber

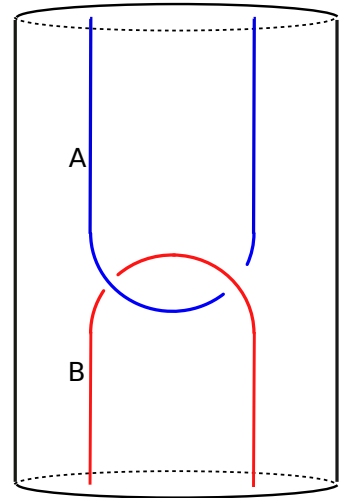

(b) Whitehead Clasp

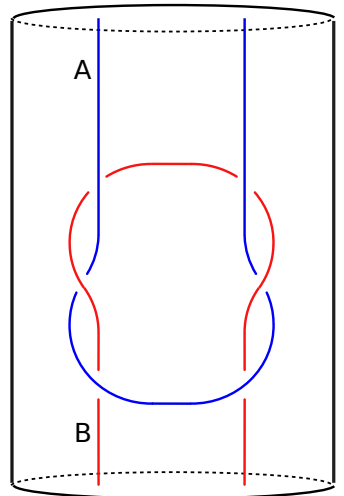

(c) Square Knot Clasp

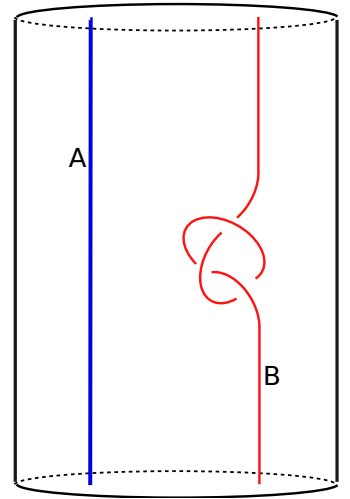

(d) Spanning Arcs

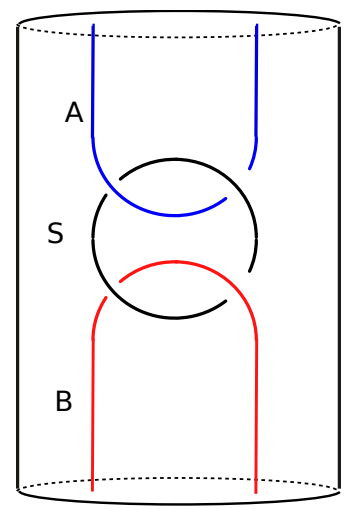

(e) Antoine Clasp

Figure 5. Clasps and Spanning Arcs

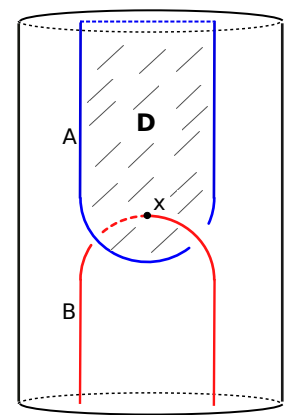

Figure 6. Whitehead Clasp with Spanning Disc

closed curves. Consider an innermost such curve in $M$. If this curves bounds a disc in $D$ missing $x$, the disc in $M$ bounded by the curve together with the disc in $D$ bounded by the curve bound a 3 ball. This 3 -ball can be used to push the disc in $M$ to the disc in $D$ which 
can then be pushed slightly off $D$. This process reduces the number of curves of intersection of $M$ with $D$.

If this innermost curve in $M$ bounds a disc in $D$ containing $x$, the disc in $M$ bounded by the curve together with the disc in $D$ bounded by the curve form a 2-sphere that is pierced once by $B$. However, this is impossible. So eventually all curves of intersection of $M$ with $D$ can be eliminated. But then $M$ misses a spanning arc of the chamber which is impossible. So no interior meridional disc $M$ can miss $A \cup B$.

The proofs for the Square Knot clasp and the Antoine clasp are similar. Note that the Antoine clasp can be divided into two Whitehead clasps by adding a meridional disc in the middle of the cylinder in Figure 5(e). Then the techniques of Theorem 4.4 below can be used to show that the geometric index is two.

\section{Main Results}

We now work towards proving the main result (Theorem 4.4) and some corollaries.

Setup: Throughout this section, let $L$ be a link in the interior of a solid torus $T$ and let $D_{0}, \ldots D_{m-1}$ be a cyclically ordered pairwise disjoint collection of meridional discs in $T$, each intersecting $L$ transversally in $n$ points. Let $C_{i}$ be the chamber bounded by $D_{i-1}$ and $D_{i \bmod m}$ for $1 \leq i \leq m$.

Remark 4.1. Note that $N\left(L \cap C_{i}, C_{i}\right) \leq n$ for each $i$. This follows from the fact that an interior meridional disc parallel to and close enough to the ends of $C_{i}$ intersects $L \cap C_{i}$ in $n$ points by the setup.

Lemma 4.2. If $N\left(L \cap C_{i}, C_{i}\right) \geq n$ for each $i$, and if $K$ is a meridional disc for $T$ that misses $\cup_{i=1}^{k} D_{i}$, then $|L \cap K| \geq n$.

Proof. This follows since any such meridional disc is an interior meridional disc for one of the chambers.

Lemma 4.3. Assume $N\left(L \cap C_{i}, C_{i}\right) \geq n$ for each $i$. Let $D^{\prime}$ be a disc that lies in the interior of $T$ with $D^{\prime} \cap\left(\cup_{i=0}^{m-1} D_{i}\right)=\partial D^{\prime}$. Then $\partial D^{\prime}$ is in some $D_{i}$. Let $D^{\prime \prime}$ be the disc bounded by $\partial D^{\prime}$ in $D_{i}$. Then $\left|L \cap D^{\prime}\right| \geq\left|L \cap D^{\prime \prime}\right|$.

Proof. Suppose, to the contrary, that $\left|L \cap D^{\prime}\right|<\left|L \cap D^{\prime \prime}\right|$. Then the meridional disc $\left(D_{i}-\right.$ $\left.D^{\prime \prime}\right) \cup D^{\prime}$ meets $L$ in fewer points than $D_{i}$. This disc can be pushed slightly off $D_{i}$ so that it still meets $L$ in fewer points than $D_{i}$. But this contradicts Lemma 4.2.

Theorem 4.4. Let $L, T$, and $D_{0}, \ldots D_{m-1}$ be as in the setup above. If any meridional disc in $T$ that misses $D=\cup_{i=0}^{i=m-1} D_{i}$ intersects $L$ in at least $n$ points, then $N(L, T)=n$. 
Proof. Since the setup implies $N(L, T) \leq n$, it suffices to show $N(L, T) \geq n$. Let $K$ be a meridional disc of $T$ so that $|K \cap L|$ is minimal. Without loss of generality, we may assume that $\partial K \cap\left(\bigcup_{i=0}^{m-1} \partial D_{i}\right)=\emptyset, \mathrm{K}$ is in general position with respect to $\mathrm{D}$, and $L \cap K \cap D=\emptyset$.

Note that if $\partial K \cap\left(\bigcup_{i=0}^{m-1} \partial D_{i}\right) \neq \emptyset$, one can use standard general position techniques to make the intersection empty. For example, use the fact that there is an isotopy of $T$ taking $K$ to a close parallel copy of any particular $D_{i}$. This isotopy can be feathered or tapered off to the identity away from a small neighborhood of the boundary of $T$ without changing the intersection of $K$ with $L$.

We show by induction on the number of components of $K \cap D$ that $|K \cap L| \geq n$. If $K \cap D=\emptyset$, then by Lemma 4.2, $|L \cap K| \geq n$. Consider a simple closed curve component $c$ of $K \cap D$ that is innermost on $\mathrm{K}$. Then $c$ bounds a disc $k$ in $K$ and a disc $d$ in some $D_{i}$. Note that $c$ may not be innermost in $D$ among the simple closed curves of $K \cap D$. However, the (possibly) singular disc $(K-k) \cup d$ meets $L$ in no more points than $K$ by Lemma 4.3. The singularities, if any, of $(K-k) \cup d$ consist of disjoint double curves that can be cut apart to give a non-singular disc $K^{\prime}$ that meets $\mathrm{L}$ in no more points than K does. See [Hem04, Ch. 4] for details on cutting apart double curves. By pushing $K^{\prime}$ slightly off $D_{i}$ we obtain a meridional disc $K^{\prime \prime}$ such that $\left|K^{\prime \prime} \cap L\right|$ is still minimal and such that $K^{\prime \prime} \cap D$ has fewer components than $K \cap D$. Therefore, by induction, $\left|K^{\prime \prime} \cap L\right| \geq n$ and so $|K \cap L| \geq n$.

Corollary 4.5. Let $L, T$, and $D_{0}, \ldots D_{m-1}$ and $C_{i}$ be as in the setup above. If $N(L \cap$ $\left.C_{i}, C_{i}\right)=n$ for each $i$, then $N(L, T)=n$.

Proof. Any meridional disc that misses $\cup_{i=0}^{i=m-1} D_{i}$ is an interior meridional disc for some chamber, and so intersects $L$ in at least $n$ points. The result now follows from Theorem 4.4 .

Corollary 4.6. Let $L, T$, and $D_{0}, \ldots D_{m-1}$ and $C_{i}$ be as in the setup above. If, for each $i$, $C_{i} \cap L$ consists of $k_{i}$ clasps and $\ell_{i}$ spanning arcs where $2 k_{i}+\ell=n$, then $N(L, T)=n$.

Proof. The hypotheses show that any interior meridional disc in $C_{i}$ intersects $L$ in at least $2 k_{i}+\ell=n$ points. (See Lemma 3.1). The result now follows from Corollary 4.5.

Corollary 4.7. Let $L, T$, and $D_{0}, \ldots D_{m-1}$ and $C_{i}$ be as in the setup above. Let $L^{\prime}$ be a link in the interior of a solid torus $T^{\prime}$ and let $D_{0}^{\prime}, \ldots D_{m-1}^{\prime}$ be a cyclically ordered collection of meridional discs in $T$, each intersecting $L^{\prime}$ transversally in $n$ points. Let $C_{i}^{\prime}$ be the region in $T^{\prime}$ bounded by $D_{i}^{\prime}$ and $D_{(i+1) \text { mod } m}^{\prime}$. If $N\left(L^{\prime}, T^{\prime}\right)=n$, and if $\left(C_{i}, C_{i} \cap L\right) \cong\left(C_{i}^{\prime}, C_{i}^{\prime} \cap L^{\prime}\right)$ for each $i$, then $N(L, T)=n$.

Proof. For each chamber $C_{i}^{\prime}, N\left(L^{\prime} \cap C_{i}^{\prime}, C_{i}^{\prime}\right)$ must be at least $n$. Otherwise, $N\left(L^{\prime}, T^{\prime}\right)$ would be less than $n$. So for each chamber $C_{i}, N\left(L \cap C_{i}, C_{i}\right)$ must be at least $n$. The result now follows from Corollary 4.5. 


\section{Examples AND Applications}

We apply the theorem and corollaries from the previous section to compute the geometric index of a number of new and old examples to illustrate the power of these results.

Figure 2 We begin with the new example(s) of Figure 2. Divide the outer torus into eight chambers as indicated in Figure 7. Each chamber has a collection of spanning arcs, and possibly Whitehead clasps, Square knot clasps, or Antoine clasps. By Lemma 3.1, the geometric index of the inner link intersected with the chamber in the chamber is 8 . The result now follows from Corollary 4.5 or from Corollary 4.6.

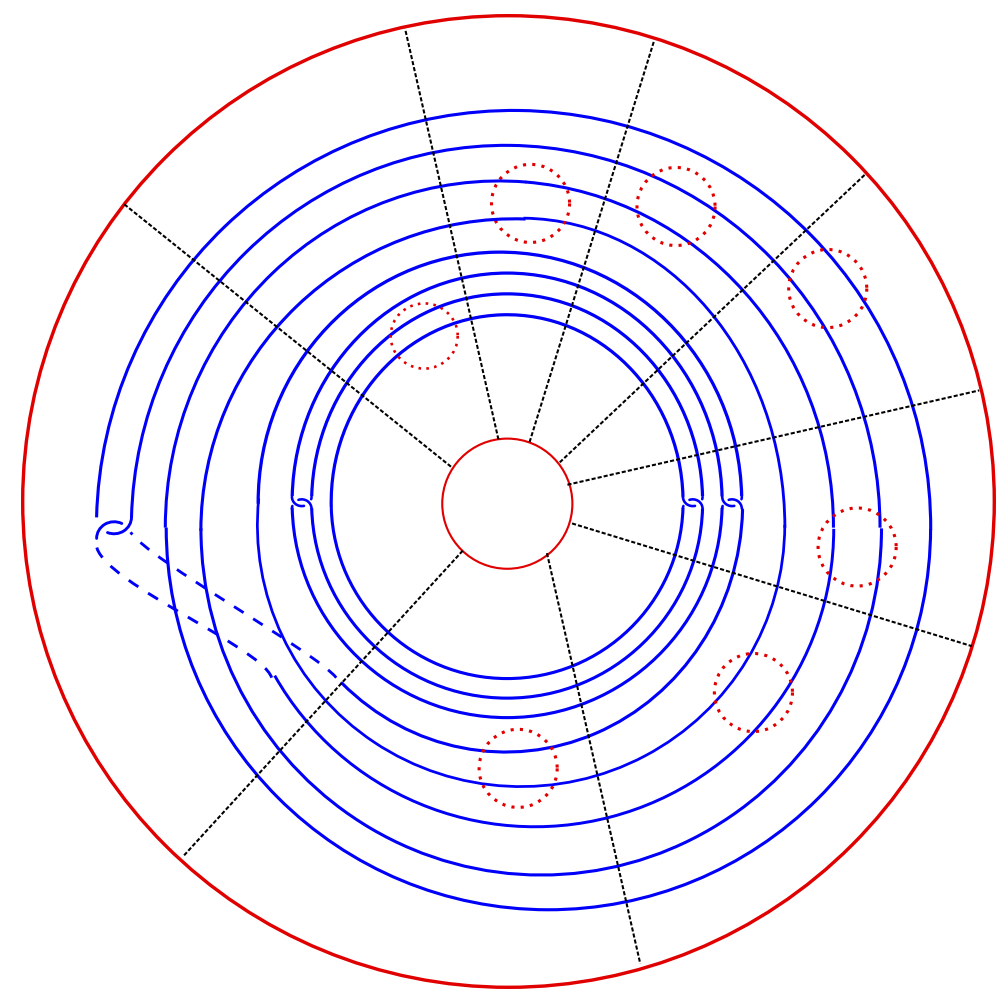

Figure 7. Chambers for Figure 2

Figures 3(a), 3(b), and 3(c): By Lemma 2.5, the geometric index of the link in each of these figures on the outer torus is 0 or 2 . So it suffices to show the geometric index is not 0 . The outer torus and link in Figure 3(c) is a covering space of the outer tori and links in Figures 3(a) and 3(b). Any meridional disc in the outer torus in Figures 3(a) and 3(b) that misses the interior link lifts to a meridional disc in the outer torus in Figure 3(c) that misses the interior link. So it suffices to show the geometric index of the link in Figure 3(c) is nonzero. There is a standard cut and paste argument that shows this. See for example [Dav07, Section 9] or [Rol90, Chapter 3-G].

An alternate approach using Corollary 4.6 is as follows. Divide the outer torus in four chambers as indicated in Figure 8(a). Each chamber has a single Whitehead clasp in it. 


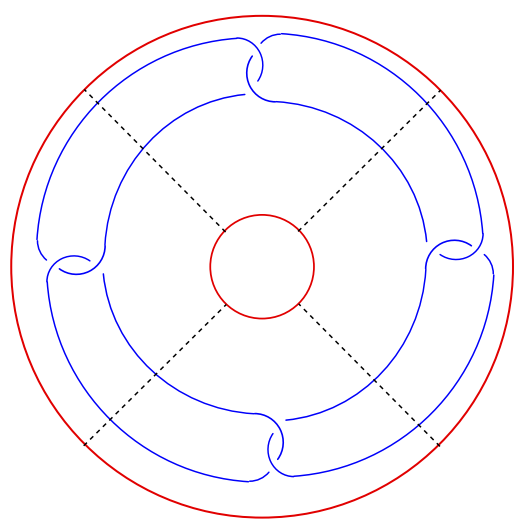

(a) Antoine Link with discs

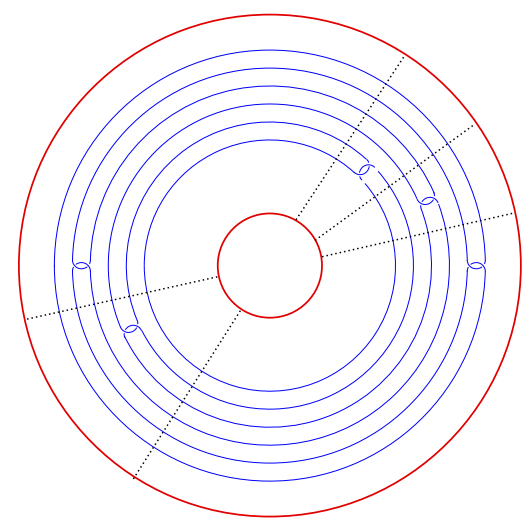

(d) Shifted Link with discs

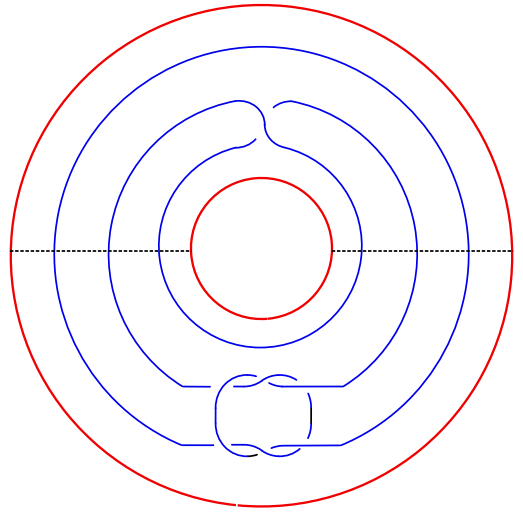

(b) Knotted Link with discs

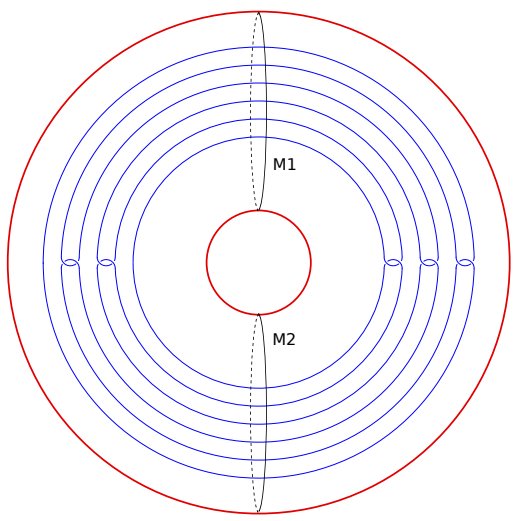

(e) Gabai Link with discs

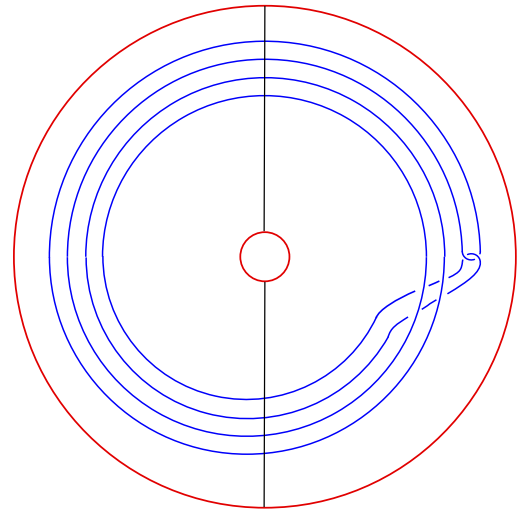

(c) McMillan Link with discs

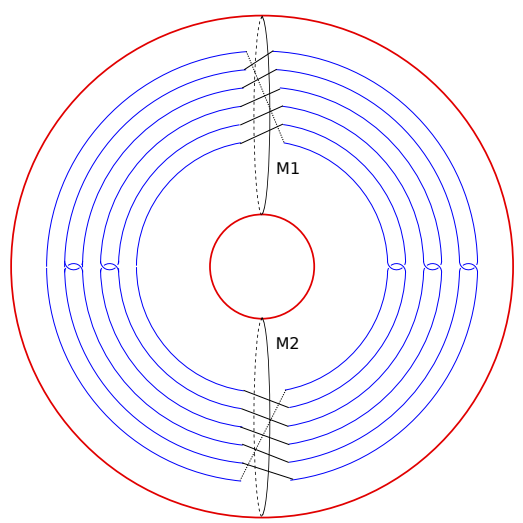

(f) Bing and Whitehead Links with discs

Figure 8. Links with Added Meridional Discs

By Lemma 3.1, the geometric index of the inner link intersected with the chamber in the chamber is 2. The result now follows from Corollary 4.5.

Figure 3(d): The algebraic index is 2 since the inner curve has winding number 2 in the outer torus. Since the geometric index is greater than or equal to the algebraic index, and since there is clearly a meridional disc that intersects the inner curve twice, the geometric index is 2 .

Figure 4(b): Divide the outer torus into two chambers as indicated in Figure 8(c). Each chamber has a Whitehead clasp and two spanning arcs or four spanning arcs. By Lemma 3.1 , the geometric index of the inner link intersected with the chamber in the chamber is 4 . The result now follows from Corollary 4.5 or from Corollary 4.6.

Figure 4(a): Divide the outer torus into two chambers as indicated in Figure 8(b). Each chamber has a Whitehead clasp and spanning arc or a Square Knot clasp and spanning arc. By Lemma 3.1, the geometric index of the inner link intersected with the chamber in the chamber is 3 . The result now follows from Corollary 4.5 or from Corollary 4.6. 
Figure 4(c): In Figure 8(d), we have slightly shifted the inner link by a homeomorphism and divided the outer torus into 5 chambers. The inner link, intersected with each chamber, consists of a Whitehead clasp and four spanning arcs. By Lemma 3.1, the geometric index of the inner link intersected with the chamber in the chamber is 6 . The result now follows from Corollary 4.5 or from Corollary 4.6.

Alternately, consider Figures 8(e) and 8(f). Corollary 4.7 applies directly since the complements of the two indicated meridional discs are homeomorphic. Figure 8(f) consists of two Bing links and a Whitehead link, so the geometric index of the inner link in the outer torus is 6. By Corollary 4.7, the geometric index of the inner link in the outer torus in Figure 8(e) is also 6 .

\section{REFERENCES}

[AS89] Fredric D. Ancel and Michael P. Starbird, The shrinkability of Bing-Whitehead decompositions, Topology 28 (1989), no. 3, 291-304, DOI 10.1016/0040-9383(89)90010-4. MR1014463 (90g:57014)

[AW] Kathryn B. Andrist and David G. Wright, On computing the geometric index, Proceedings of the seventeenth annual workshop in geometric topology (Colorado College, June 15-17, 2000), F. Tinsley, Ed., Colorado College, Colorado Springs, 2000, pp. 35-38.

[Bro61] Morton Brown, The monotone union of open n-cells is an open n-cell, Proc. Amer. Math. Soc. 12 (1961), 812-814. MR0126835 (23 \#A4129)

[Dav07] Robert J. Daverman, Decompositions of manifolds, AMS Chelsea Publishing, Providence, RI, 2007. Reprint of the 1986 original. MR2341468 (2008d:57001)

[DV09] Robert J. Daverman and Gerard A. Venema, Embeddings in manifolds, Graduate Studies in Mathematics, vol. 106, American Mathematical Society, Providence, RI, 2009. MR2561389 (2011g:57025)

[Gab11] David Gabai, The Whitehead manifold is a union of two Euclidean spaces, J. Topol. 4 (2011), no. 3, 529-534, DOI 10.1112/jtopol/jtr010. MR2832566 (2012i:57037)

[GRW17] Dennis J. Garity, Dušan D. Repovš, and David G. Wright, Contractible 3-manifolds and the Double 3-Space Property, Trans. Amer. Math. Soc. 370 (2017), no. 3, 2039-2055, DOI 10.1090/tran/7035. MR3739201

[GRWŽ11] Dennis Garity, Dušan Repovš, David Wright, and Matjaž Željko, Distinguishing Bing-Whitehead Cantor sets, Trans. Amer. Math. Soc. 363 (2011), no. 2, 1007-1022, DOI 10.1090/S0002-99472010-05175-X. MR2728594 (2011j:54034)

[Hem04] John Hempel, 3-manifolds, AMS Chelsea Publishing, Providence, RI, 2004. Reprint of the 1976 original. MR2098385 (2005e:57053)

[McM62] D. R. McMillan Jr., Some contractible open 3-manifolds, Trans. Amer. Math. Soc. 102 (1962), 373-382. MR0137105 (25 \#561)

[Mye88] Robert Myers, Contractible open 3-manifolds which are not covering spaces, Topology 27 (1988), no. 1, 27-35, DOI 10.1016/0040-9383(88)90005-5. MR935526 (89c:57012)

[Mye99] _ Contractible open 3-manifolds which non-trivially cover only non-compact 3-manifolds, Topology 38 (1999), no. 1, 85-94, DOI 10.1016/S0040-9383(98)00004-4. MR1644087 (99g:57022)

[Rol90] Dale Rolfsen, Knots and links, Mathematics Lecture Series, vol. 7, Publish or Perish, Inc., Houston, TX, 1990. Corrected reprint of the 1976 original. MR1277811 (95c:57018)

[RS82] Colin P. Rourke and Brian J. Sanderson, Introduction to piecewise-linear topology, Springer Study Edition, Springer-Verlag, Berlin-New York, 1982. Reprint. MR665919 (83g:57009)

[Sch53] Horst Schubert, Knoten und Vollringe, Acta Math. 90 (1953), 131-286 (German). MR0072482 (17,291d) 
[Whi35] J. H. C. Whitehead, A certain open manifold whose group is unity, Quart. J. Math. 6 (1935), no. 6, 268-279.

[Wri89] David G. Wright, Bing-Whitehead Cantor sets, Fund. Math. 132 (1989), no. 2, 105-116. MR1002625 (90d:57020)

[Wri92] _ Contractible open manifolds which are not covering spaces, Topology 31 (1992), no. 2, 281-291, DOI 10.1016/0040-9383(92)90021-9. MR1167170 (93f:57004)

Mathematics Department, Utah Valley University, Orem, Utah 84058

E-mail address: kathy.andrist@uvu.edu

$U R L:$ http://www.uvu.edu/profpages/profiles/show/user_id/146

Mathematics Department, Oregon State University, Corvallis, OR 97331, U.S.A.

E-mail address: garity@math.oregonstate.edu

$U R L:$ http://www.math.oregonstate.edu/〜garity

Faculty of Education, and Faculty of Mathematics and Physics, University of Ljubljana \& Institute of Mathematics, Physics and Mechanics, Luubluana, SI-1000, Slovenia

E-mail address: dusan.repovs@guest.arnes.si

$U R L:$ http://www.fmf.uni-lj.si/ ^repovs

Department of Mathematics, Brigham Young University, Provo, UT 84602, U.S.A.

E-mail address: wright@math.byu.edu

$U R L:$ http://www. math. byu.edu/〜wright 